\title{
Diagnóstico y tratamiento de la cardiopatía isquémica en mujeres
}

\author{
Mónica Jaramillo-Jaramillo ${ }^{\mathrm{a}, \mathrm{b}, *}$ y Juan M. Zambrano-Chaves ${ }^{\mathrm{a}, \mathrm{b}}$
}

a Hospital Universitario Fundación Santa Fe de Bogotá, Bogotá, Colombia
b Facultad de Medicina, Universidad de los Andes, Bogotá, Colombia

Recibido el 13 de octubre de 2017; aceptado el 24 de noviembre de 2017

Disponible en Internet el 10 de enero de 2018

\section{PALABRAS CLAVE \\ Diagnóstico; \\ Cardiopatía \\ isquémica; \\ Tratamiento; \\ Mujer}

\begin{abstract}
Resumen La enfermedad isquémica del corazón representa una de las principales causas de mortalidad en Colombia y en el mundo. Su impacto sobre la morbilidad y mortalidad ha favorecido un importante esfuerzo global para investigar en esta área. Este último ha permitido identificar diferencias en múltiples aspectos de la enfermedad teniendo en cuenta una perspectiva del sexo. En este artículo se examinan diferencias en cuanto a fisiopatología, diagnóstico y tratamiento de la enfermedad coronaria en mujeres.

( 2017 Publicado por Elsevier España, S.L.U. en nombre de Sociedad Colombiana de Cardiología y Cirugía Cardiovascular. Este es un artículo Open Access bajo la licencia CC BY-NC-ND (http:// creativecommons.org/licenses/by-nc-nd/4.0/).
\end{abstract}

\section{Diagnosis and treatment of ischemic cardiomyopathy in women}

Abstract Ischemic heart disease is one of the main causes of mortality in Colombia as well as worldwide. Its impact on morbidity and mortality has favored an important global effort to conduct research in this area. As a result, differences in multiple aspects of this disease regarding gender differences have surfaced. This paper examines differences in pathophysiology, diagnosis and treatment of coronary disease in women.

(c) 2017 Published by Elsevier España, S.L.U. on behalf of Sociedad Colombiana de Cardiología y Cirugía Cardiovascular. This is an open access article under the CC BY-NC-ND license (http: / / creativecommons.org/licenses/by-nc-nd/4.0/).

\footnotetext{
* Autor para correspondencia.

Correo electrónico: mjaramil@uniandes.edu.co (M. Jaramillo-Jaramillo).
} 


\section{Introducción y epidemiología}

Las patologías que hacen parte del grupo de enfermedades cardiovasculares, se encuentran entre las primeras causas de muerte. La enfermedad isquémica del corazón, que comprende la angina pectoris, la falla cardiaca isquémica y el infarto agudo de miocardio, se registró como la primera causa de muerte en el mundo en 2010 .

En Colombia la enfermedad isquémica del corazón fue la primera causa de muerte en el conjunto de las enfermedades del sistema circulatorio, representando el 49,5\% de las muertes en este grupo ${ }^{1}$.

Tradicionalmente se ha considerado que la enfermedad isquémica del corazón es una patología que afecta principalmente a los hombres. De acuerdo con el informe sobre enfermedades crónicas del Instituto Nacional de Salud de Colombia, los hombres tienen mayor riesgo de desarrollar y morir por enfermedad isquémica del corazón en comparación con las mujeres ${ }^{1}$. Esta relación se ha mantenido a través de los años; para 2014 alcanzó una razón de tasas de incidencia hombre:mujer de 1,74.

En mujeres, en el informe de 2010-2014 de incidencia y mortalidad de enfermedad isquémica del corazón en Colombia, el promedio anual de casos nuevos fue de 52.948. La tasa de incidencia corregida varió en el periodo 2010-2014, aumentó de 257 por 100.000 habitantes al inicio del periodo, hasta 280 por 100.000 habitantes en 2014 , representando en términos absolutos un incremento de 23 casos nuevos por cada 100.000 habitantes. De manera contraria se observó una disminución leve en la tasa de mortalidad, que decreció de 67,6 por 100.000 habitantes en 2010 a 66,3 en 2014.

En este artículo se expondrán diferencias en cuanto a fisiopatología, diagnóstico y tratamiento de la cardiopatía isquémica con énfasis en mujeres, comparadas con su contraparte masculina.

\section{Fisiopatología de la enfermedad arterial coronaria}

Décadas de investigación sobre la fisiopatología de la enfermedad de las arterias coronarias han provisto conocimientos en factores específicos por género que son importantes en el diagnóstico no invasivo de síndromes de isquemia miocárdica en mujeres.

La evidencia conseguida en el WISE (Womenś Ischemic Syndrome Evaluation) ${ }^{2}$ indica que la enfermedad de las arterias coronarias en las mujeres se extiende más allá de las estenosis ateroscleróticas en las arterias coronarias epicárdicas hasta incluir disfunción de la microvasculatura coronaria. Condiciones adicionales que contribuyen al espectro fisiopatológico completo de la enfermedad isquémica del corazón en mujeres incluyen el vasoespasmo coronario y la disección de arterias coronarias, que afecta principalmente las arterias coronarias epicárdicas y se da en mujeres jóvenes. Las implicaciones de estas condiciones diversas son sustanciales, crean retos diagnósticos y limitaciones en la aplicación de las estrategias tradicionales que son adecuadas para detectar enfermedad de las arterias coronarias epicárdicas obstructivas, pero insuficientes para el estudio de muchas mujeres con dolor torácico ${ }^{3}$.
Considerando el espectro de la enfermedad isquémica del corazón, la investigación más allá de las pruebas estándares de estrés, frecuentemente es necesaria para definir la etiología de los síntomas en las mujeres. Estudios recientes han demostrado que la disfunción de las arteriolas coronarias más pequeñas puede causar isquemia crónica, infarto agudo de miocardio (IAM) o miocardiopatía inducida por estrés ${ }^{4}$.

En 2014 la Asociación Americana del Corazón, con base en las potenciales diferencias entre los sexos en fisiopatología y presentación clínica de la enfermedad isquémica del corazón en mujeres ${ }^{5}$, hizo una publicación en la que se sugiere un nuevo algoritmo teniendo en cuenta la eficacia y seguridad del tratamiento médico óptimo y la revascularización así como la evidencia clínica y el significado pronóstico de la enfermedad de las arterias coronarias no obstructiva detectada por ultrasonido o tomografía axial computarizada de arterias coronarias (TACAC). Esta declaración se enfoca en dos patrones de presentación clínica y la carga de enfermedad correlativa: 1 . Isquemia inducible causada por una enfermedad de las arterias coronarias con estenosis significativa, y 2 . Identificación de la extensión y severidad de la isquemia miocárdica que resulta de disfunción vascular coronaria en el caso de enfermedad de las arterias coronarias no obstructiva. Pese a que las mujeres con síntomas isquémicos estables, tienen mayor prevalencia de enfermedad de las arterias coronarias no obstructiva, poseen mayor riesgo de eventos coronarios en comparación con la población general. La isquemia miocárdica se asocia con mayor mortalidad por enfermedad isquémica del corazón en mujeres sintomáticas. El concepto de que los síntomas en las mujeres están correlacionados con disfunción vascular coronaria en el contexto de remodelación vascular expansiva y placas no obstructivas, es un componente crítico para entender los patrones femeninos específicos en la presentación de los síntomas y el riesgo elevado de enfermedad isquémica del corazón. Así, hoy es fundamental documentar isquemia miocárdica y detectar enfermedad de las arterias coronarias para determinar el riesgo de enfermedad isquémica del corazón y guiar las decisiones terapéuticas. Por tanto, las mujeres con enfermedad de las arterias coronarias no obstructiva y anormalidades en la prueba de estrés, no se deben definir como un resultado falso positivo, sino informar como anormal y clasificarlas con un riesgo elevado de enfermedad isquémica del corazón.

\section{Presentación clínica}

La presentación más común de isquemia miocárdica es dolor o molestia torácica; no obstante, las mujeres tienen un patrón y una distribución diferentes de los síntomas no relacionados con dolor torácico. Respecto a los hombres, los síntomas son más precipitados por estrés emocional o mental y menos por ejercicio físico.

No se han encontrado síntomas específicos por género. Las mujeres informan más molestias epigástricas y náuseas asociadas, irradiación a brazos, hombros o región interescapular. El registro WISE refiere que las mujeres tienen un amplio rango de síntomas y muchos de ellos en reposo, entre los cuales se enumeran disnea, cansancio, mareo, sensación de miedo o pánico, indigestión, palpitaciones, debilidad generalizada o síntomas que asemejan un resfriado ${ }^{2}$. 


\section{Pruebas invasivas y no invasivas}

\section{Electrocardiograma de ejercicio}

Una prueba de ejercicio es la recomendación inicial del AHA para mujeres sintomáticas con riesgo intermedio que pueden hacer ejercicio. La exactitud total de esta prueba es modesta, y la sensibilidad y especificidad de la desviación del segmento ST durante el ECG de ejercicio es menor en mujeres que en hombres. Las sensibilidades y especificidades medias reportadas del ECG de ejercicio son respectivamente 61 y $70 \%$ para mujeres y 72 y $77 \%$ para hombres $^{6}$. El valor predictivo positivo (VPP) de la depresión durante el ECG de ejercicio es más bajo en mujeres que en hombres $(47 \% \text { vs. } 77 \%, p<0,05)^{6}$. No se conocen las causas de la baja exactitud en las mujeres, pero se cree que la capacidad de ejercicio reducida, la atenuación por la pared torácica y los efectos de los estrógenos podrían potenciar los cambios en el ST. El uso de un nomograma específico para mujeres para evaluar la capacidad máxima de ejercicio también es útil, y es un predictor independiente de muerte ${ }^{7}$.

A pesar del relativamente bajo VPP de la prueba de estrés convencional, su alto valor predictivo negativo (aproximadamente $80 \%$ ) es valioso para descartar enfermedad de las arterias coronarias obstructiva y predecir sobrevida libre de eventos. Por este motivo, de acuerdo con la AHA para mujeres con capacidad de ejercicio adecuada y ECG de reposo normal, el ECG de ejercicio permanece como la recomendación inicial ${ }^{5}$.

\section{Pruebas de estrés con imágenes}

Las pruebas de estrés con imágenes de ecocardiograma o perfusión miocárdica con isonitrilos se recomiendan para la evaluación inicial de mujeres sintomáticas con riesgo intermedio o alto de enfermedad de las arterias coronarias quienes tienen anormalidades del segmento ST en el ECG, pobre capacidad de ejercicio, o un ECG de ejercicio anormal (de intermedio a alto riesgo). Las imágenes de estrés proveen un valor incrementado sobre el ECG en reposo, el ECG de ejercicio, y la evaluación clínica del riesgo.

\section{Ecocardiografía}

El ecocardiograma permite la evaluación visual de la función sistólica, de anormalidades de la movilidad de la pared inducidas por estrés y de cicatrices miocárdicas. También puede identificar otras causas de angina no relacionadas con enfermedad de las arterias coronarias, como valvulopatías, disección o aneurisma aórtico, hipertensión pulmonar y enfermedad pericárdica. El desempeño de la ecocardiografía de estrés es superior al ECG de ejercicio. Se reporta sensibilidad y especificidad promedio de 79 y $83 \%$, en mujeres ${ }^{8}$. Al no usar radiación ionizante, es la principal alternativa en mujeres jóvenes o embarazadas.

\section{Imágenes de perfusión miocárdica (IPM) con estrés}

La detección de defectos de perfusión durante el reposo y el estrés con SPECT y PET son la piedra angular del diagnóstico con IPM. Ambas modalidades permiten evaluar la motilidad regional y la fracción de eyección del ventrículo izquierdo. Hay evidencia amplia que las IPM ofrecen una mejor exactitud diagnóstica que el ECG de ejercicio en mujeres. En la IPM por SPECT con las técnicas de corrección y atenuación y el uso de radiofármacos con Tc 99, el desempeño en mujeres es similar al ecocardiograma estrés. En un metaanálisis que evalúa las diferencias de sexo en IPM con SPECT, no hubo diferencias significativas entre hombres y mujeres (sensibilidad y especificidad, 84 y $79 \%$ en mujeres versus 89 y $71 \%$ en hombres).

Las IPM mediante PET tienen mejor desempeño que las obtenidas por SPECT por disminuir la atenuación producida por los tejidos blandos, lo cual beneficia a las mujeres. Adicionalmente, las IPM obtenidas por PET pueden cuantificar el flujo sanguíneo miocárdico (FSM) en reposo y ejercicio, así como la reserva de flujo coronario (RFC), las cuales pueden confirmar o aumentar la detección de isquemia, considerando que una RFC menor de 2 es sugestiva de disfunción vascular ${ }^{9}$. Una RFC baja $(<1,6)$ medida por PET se ha asociado con muerte cardiovascular y admisiones por insuficiencia cardiaca independiente del puntaje de la angiografía invasiva $^{10}$. La RFC puede reflejar una medida integrada de enfermedad de las arterias coronarias o enfermedad microvascular. Para las mujeres, en quienes la enfermedad no obstructiva y la enfermedad microvascular pueden ser más prevalentes, estas mediciones cuantitativas pueden ayudar a identificar enfermedad que podría no ser detectada por medio de modalidades diagnósticas tradicionales. Más allá de esto, la medición cuantitativa de flujo puede ayudar a identificar pacientes de alto riesgo, cambiar paradigmas en las mujeres y dilucidar mecanismos que expliquen la discrepancia relativa entre los resultados clínicos en mujeres y la severidad de enfermedad obstructiva de las arterias coronarias.

\section{Resonancia magnética cardiaca}

En el momento el uso de pruebas de estrés con imágenes con resonancia magnética (RM) en mujeres sintomáticas con riesgo intermedio a alto de enfermedad de las arterias coronarias, está considerado como recomendación clase Ilb en las guías de la $\mathrm{AHA}^{5}$. Las imágenes con RM tienen una resolución espacial superior y mayor detección de isquemia subendocárdica ${ }^{5}$, especialmente en mujeres con cambios en el ECG y coronariografía invasiva normal. En el futuro se podrá usar por su capacidad para evaluar el flujo sanguíneo miocárdico.

\section{Angiografía coronaria}

\section{Angiografía coronaria por tomografía computarizada (ACTC)}

De acuerdo con las recomendaciones de la AHA, la ACTC "puede ser razonable"' en mujeres sintomáticas con riesgo intermedio de enfermedad de las arterias coronarias, incluyendo aquellas con resultados de exámenes inexactos ${ }^{5}$.

La detección de calcio coronario en imágenes de TC confiere alta sensibilidad para enfermedad de las arterias coronarias por angiografía invasiva (sensibilidad reportada 
$\geq 96 \%$ y es un predictor independiente para riesgo cardiovascular en hombres y mujeres. Sin embargo, el calcio coronario no es altamente específico para enfermedad de las arterias coronarias (50 a 66\%); no se correlaciona bien con la severidad y localización de estenosis ${ }^{11}$. La ausencia de calcio en la imagen de TC indica una probabilidad baja de enfermedad obstructiva de las arterias coronarias, pero no descarta la posibilidad de placa no calcificada. Esto puede ser relevante en mujeres quienes tienen puntajes de calcio más bajos que los hombres ${ }^{12}$. La diferencia en la composición de placas puede explicar parcialmente la variabilidad en la presentación clínica y los resultados de la $\mathrm{ECl}$ en ambos sexos. La incapacidad del puntaje de calcio para descartar las placas no calcificadas, sumado a la irradiación, le resta valor diagnóstico, principalmente en mujeres jóvenes y embarazadas 6 .

La exactitud de la ACTC es elevada para la detección de enfermedad obstructiva de las arterias coronarias. El estudio ACCURACY ${ }^{13}$, reportó sensibilidad y especificidad similar entre sexos ( 90 y $89 \%$ en mujeres versus 96 y $78 \%$ en hombres, respectivamente).

El VPN alto de la angiografía por TC (91 a 100\% en mujeres) la hace muy útil para descartar enfermedad de las arterias coronarias y predecir resultados clínicos favorables. En pacientes con angiografías coronarias por TC normales la mortalidad es baja; varía entre 0,15 a 0,4\%. La ACTC puede predecir resultados en mujeres y hombres; en una cohorte prospectiva con más de 12.000 mujeres la mortalidad aumentó proporcionalmente con el número de vasos enfermos y la severidad de la estenosis ${ }^{14}$. La razón ajustada de riesgo para toda causa de mortalidad en enfermedad de tres vasos comparada con una arteriografía normal fue más alta en mujeres que en hombres (RR 4,21 vs. 3,27). Mientras que la enfermedad de las arterias coronarias obstructiva confiere para riesgo de mortalidad mayor tanto para mujeres como hombres, la enfermedad de las arterias coronarias no obstructiva ( $<50 \%$ de estenosis) confiere una mortalidad más alta solo en mujeres ${ }^{15}$. Estas últimas presentan mayor mortalidad que los hombres a pesar de una enfermedad menos extensa. La TC con IPM con energía dual o técnica de adquisición dinámica, y las mediciones de reserva de flujo, pueden mejorar potencialmente la exactitud diagnóstica de la angiografía con $\mathrm{TC}^{16}$.

\section{Angiografía coronaria invasiva}

La anatomía coronaria y la enfermedad obstructiva de las arterias coronarias también se evalúan mediante angiografía coronaria invasiva $(\mathrm{ACl})$, considerada la referencia estándar para el diagnóstico de enfermedad obstructiva de las arterias coronarias epicárdicas. A pesar de la relación ampliamente conocida entre la enfermedad de las arterias coronarias y la isquemia miocárdica, una proporción de pacientes con signos y síntomas sugestivos de isquemia miocárdica no tiene lesiones en la $\mathrm{ACl}$ que limiten significativamente el flujo. La sensibilidad de la $\mathrm{ACl}$ puede ser sorprendentemente baja; el $40 \%$ de las mujeres con sospecha de enfermedad de las arterias coronarias tiene arterias coronarias angiográficamente normales o casi normales ${ }^{17}$.

Las anormalidades en la microcirculación coronaria podrían ser la causa de síntomas sugestivos de enfermedad de las arterias coronarias. En las dos últimas décadas, la disfunción microvascular coronaria (DMC) se ha establecido como una entidad fisiopatológica independiente. Los estudios han mostrado que las anormalidades estructurales y funcionales inherentes de la microcirculación pueden alterar la perfusión miocárdica y causar isquemia ${ }^{18}$. Debido a limitaciones en la resolución espacial, los sistemas de angiografía invasivos y no invasivos convencionales sólo pueden visualizar la anatomía de las arterias coronarias epicárdicas, limitando su visualización directa en vivo.

La evaluación de la microcirculación coronaria actualmente es considerada como la referencia estándar para el diagnóstico de enfermedad microvascular ${ }^{19}$. La prueba más recomendada para evaluar la microcirculación es la medición de la reserva de flujo coronario ${ }^{20}$. Hoy, la disfunción microvascular coronaria se considera la base fisiopatológica para la presencia de angina, remodelación miocárdica y resultados adversos como muerte y eventos cardiovasculares mayores en pacientes con enfermedad de las arterias coronarias estable y síndrome coronario agudo ${ }^{20}$, por lo que su detección y evaluación podrían ser rutinarias en el futuro.

\section{Tratamiento de la enfermedad coronaria}

Las mujeres reciben menores terapias para prevenir y tratar la $\mathrm{ECl}$ a pesar de las guías que muestran su beneficio. Las mujeres tienen menor tamizaje de colesterol, menores terapias para disminuir el colesterol, menor uso de heparina, betabloqueadores y aspirina durante el infarto agudo de miocardio, menos tratamiento antiplaquetario en prevención secundaria, menores remisiones a rehabilitación cardiaca y menor número de cardiodesfibriladores implantables (CDI) y trasplantes cardiacos al compararlos con los hombres con las mismas indicaciones reconocidas ${ }^{21}$.

\section{Tratamiento del infarto agudo de miocardio}

Las mujeres reciben con menor frecuencia el tratamiento adecuado durante un infarto agudo de miocardio que los hombres a pesar de haberse demostrado beneficios en la mortalidad. Ellas tienen peores desenlaces que los hombres ya sea con trombólisis o con intervención percutánea (IPC), pero esto se debe a factores de confusión. Las mujeres tienen un resultado más favorable con IPC comparado con terapia trombolítica en el contexto de infarto agudo de miocardio con elevación del ST y se benefician de una estrategia invasiva temprana en presencia de infarto agudo de miocardio sin elevación del ST.

\section{Reperfusión y revascularización en infarto agudo de miocardio con elevación del ST}

\section{Tratamiento trombolítico}

La trombólisis oportuna reduce la mortalidad independientemente de la edad o el sexo. Se recomienda en pacientes sin contraindicaciones quienes consultan a un hospital sin capacidad de realizar IPC temprana y/o cuando ésta puede tardar más de 120 minutos desde el primer contacto médico 
(clase I, grado de evidencia A). Las mujeres tratadas con trombólisis tienen mayor morbilidad y mortalidad, fenómeno que se explica parcialmente por el mayor número de comorbilidades, más complicaciones no fatales como choque, mayor riesgo de reinfarto, tratamiento menos agresivo y mayor mortalidad. La enoxaparina como terapia adjunta reduce la muerte a los 30 días, a expensas de aumento en el riesgo de sangrado. El sexo femenino es un factor de riesgo independiente de sangrado intracraneano con el tratamiento trombolítico, sumado a que las mujeres tienen mayor número de contraindicaciones relativas como edad avanzada, hipertensión arterial y tamaño corporal pequeño. A pesar de esto, los trombolíticos son benéficos y han mostrado disminuir la mortalidad significativamente si se usan en las primeras 12 horas en pacientes sin contraindicaciones e infarto agudo de miocardio sin elevación del $\mathrm{ST}^{22}$.

\section{IPC primaria}

En un análisis de 22 estudios que aleatorizaron 6.763 pacientes con infarto agudo de miocardio con elevación del ST a IPC primaria versus trombolíticos, encontraron que las mujeres tuvieron menor mortalidad a 30 días con IPC primaria, sin importar si se realizó en las primeras dos horas o después de dos horas ${ }^{23}$. Es importante resaltar la mortalidad elevadísima en mujeres que consultan tarde y son tratadas con terapia trombolítica. El uso de angioplastia primaria elimina virtualmente el riesgo de hemorragia intracraneana y es un predictor independiente de sobrevida en mujeres ${ }^{24}$. Aunque se disminuye el sangrado intracraneano, las mujeres tienen mayor número de eventos de sangrado no cerebrales, complicaciones vasculares y necesidad de transfusión sanguínea ${ }^{25}$.

\section{Revascularización en infarto agudo de miocardio sin elevación del ST}

Las mujeres con infarto agudo de miocardio sin elevación del ST tienen más complicaciones que los hombres, incluidos sangrado, insuficiencia cardiaca, choque, insuficiencia renal, reinfarto, ictus y readmisión.

Una estrategia invasiva temprana es clase I, grado de evidencia $A$ en mujeres con alto riesgo. Esta recomendación se basa en varios estudios que demuestran una reducción significativa en muertes, infarto agudo de miocardio al año y rehospitalización en mujeres con estrategia invasiva en mujeres con hallazgos de alto riesgo ${ }^{26}$.

\section{Tratamiento farmacológico}

Las metas de la farmacoterapia son reducir la morbilidad y mortalidad, prevenir complicaciones y mejorar la calidad de vida. De similar importancia después de un infarto agudo de miocardio es la descontinuación de medicamentos potencialmente peligrosos o que no las beneficien. $\mathrm{El}$ tratamiento hormonal con estrógenos más progesterona o estrógenos solos no está indicado en la mujer postmenopáusica después del infarto. Si la mujer desea la terapia con hormonas, se le debe explicar claramente el aumento de riesgo de eventos cardiovasculares (tabla 1).

\section{Tratamiento a largo plazo de la enfermedad de las arterias coronarias}

\section{Manejo farmacológico}

En la actualidad existen cuatro familias de medicamentos recomendados en el manejo crónico de la cardiopatía

Tabla 1 Terapia farmacológica en infarto agudo de miocardio

\begin{tabular}{l} 
Terapia \\
\hline Ácido acetil \\
salicílico \\
Clopidogrel \\
\\
Prasugrel y \\
ticagrelor \\
Inhibidor \\
glicoproteín \\
Ilb/Illa
\end{tabular}

Anticoagulantes

Betabloqueadores

IECA/ARA

Estatinas

Efecto

$\downarrow$ mortalidad vascular $16 \%$

$\downarrow$ riesgo cardiovascular total 20 a $39 \%$ (solo infarto agudo de miocardio)

No interacción significativa con el sexo

Mujeres con peores resultados; si se estratifica por nivel de troponina no hay diferencias entre sexos

$\downarrow$ complicaciones trombóticas durante la ICP* en infarto agudo de miocardio sin elevación del ST o infarto agudo de miocardio con elevación del ST, sin importar la estrategia de revascularización Eficacia demostrada con heparina no fraccionada, heparina de bajo peso molecular y bivalirudina (infarto agudo de miocardio sin elevación del ST) $\downarrow$ mortalidad 21\%, $\downarrow$ muerte súbita $30 \%, y \downarrow$ reinfarto $25 \%$. No usar betabloqueadores no selectivos por el riesgo de vasoespasmo

Los IECA mejoran la sobrevida y atenúan la dilatación ventricular izquierda. Los ARA han demostrado no ser inferiores a los IECA. Mujeres con baja representación en los estudios. Metaanálisis mostró que el beneficio fue igual en hombres y mujeres sintomáticos, pero no en mujeres asintomáticas

$\downarrow$ mortalidad por enfermedad de las arterias coronarias $43 \%$ y $\downarrow$ reinfarto $57 \%$

Adaptada de: Boersma E. Primary coronary angioplasty vs. thrombolysis g. does time matter? a pooled analysis of randomized clinical trials comparing primary percutaneous coronary intervention and in-hospital fibrinolysis in acute myocardial infarction patients. Eur Heart J. 2006;27(7):779-88). IPC = intervención coronaria percutánea. IECA = inhibidor de la enzima convertidora de angiotensina ARA = antagonistas de los receptores de angiotensina. 
isquémica: el ácido acetilsalisílico (ASA), beta bloqueadores, inhibidores de la enzima convertidora de angiotensina (IECA/antagonistas del receptor de angiotensina 2 -ARA2) y estatinas. Se han descrito diferencias en la administración de dichos medicamentos en cuanto al sexo, en todos los casos con mayor uso en los hombres ${ }^{27-29}$. Al evaluar la adherencia a las guías en cuanto al género, se ha descrito menor adherencia en la prescripción a la misma en las mujeres, en particular ancianas ${ }^{30}$. Estos medicamentos están indicados en todas las pacientes con isquemia demostrada así no se demuestren lesiones significativas en las coronarias epicárdicas, se haya o no realizado revascularización. En caso de persistencia de síntomas, las guías indican que el tratamiento médico óptimo debe incluir al menos un medicamento para aliviar los síntomas o la isquemia además de los medicamentos para prevenir nuevos eventos. Los medicamentos de primera línea son nitratos de corta acción, así como betabloqueadores y/o calcioantagonistas para controlar la frecuencia cardiaca y los síntomas. Como medicamentos de segunda línea se recomienda agregar nitratos de larga acción o ivabradina o nicorandil o ranolazina de acuerdo con la frecuencia cardiaca, presión arterial y tolerancia ${ }^{31}$. Intervenciones dirigidas específicamente hacia las mujeres han mostrado aumentar la adherencia a la prescripción y uso de dichos medicamentos de prevención secundaria ${ }^{32}$.

\section{Dispositivos}

El CDI está recomendado en cardiopatía isquémica (al menos 6 semanas después de un infarto agudo de miocardio) para reducir la muerte súbita en pacientes con falla cardiaca sintomática (clase funcional NYHA II-IV), fracción de eyección del ventrículo izquierdo $\leq 35 \%$ después de $\geq 3$ meses de terapia médica óptima quien se espera que sobreviva por al menos 1 año con un buen estado funcional ${ }^{33}$.

\section{Revascularización quirúrgica}

El uso de derivación aortocoronaria (CABG) es menor en mujeres que en hombres. Adicionalmente, múltiples estudios han encontrado una asociación entre sexo femenino y mayor tasa de mortalidad a corto y largo plazo en mujeres sometidas a CABG, posiblemente en relación con mayor edad en el momento de presentación y comorbilidades tales como diabetes mellitus, dislipidemia, falla cardiaca congestiva y enfermedad arterial periférica ${ }^{34}$. Las diferencias en mortalidad se mantienen diez años después de realizada la intervención. Un estudio reciente sugiere que el uso de CABG adicionada al manejo médico tiene beneficios en cuanto a reducción de mortalidad por todas las causas ${ }^{35}$, aunque únicamente incluyeron $12 \%$ de mujeres dentro de su población a estudio, por lo que es importante diseñar futuros estudios que analicen el beneficio de esta intervención en esta población que ha demostrado ser más vulnerable a los desenlaces desfavorables de la enfermedad de las arterias coronarias.

\section{Conclusiones}

La enfermedad isquémica del corazón es una entidad cuya prevalencia cobra importancia comprometiendo cada año la vida de miles de colombianos. Se han descrito diferencias en presentación clínica, diagnóstico y tratamiento de la misma en cuanto al sexo. Infortunadamente, en múltiples estudios recientes se han encontrado diferencias en el manejo que desfavorecen a las mujeres, pese a que evidencia muestra el beneficio de una terapia tanto en hombres como en mujeres. Lo anterior confirma la importancia de reforzar las políticas públicas para la detección, el diagnóstico y el tratamiento eficaz de la enfermedad isquémica del corazón en mujeres para lograr una disminución de la morbimortalidad femenina.

\section{Conflicto de intereses}

Ninguno.

\section{Bibliografía}

1. Valencia-Hernández C, Vargas-Sandoval G, Castañeda-Orjuela C. Carga de enfermedad isquémica del corazón en Colombia. Carga de enfermedad por Enfermedades Crónicas No Transmisibles y Discapacidad en Colombia. V ed. Bogotá, Colombia: Imprenta Nacional de Colombia; 2015.

2. Bairey Merz CN, Shaw LJ, Reis SE, Bittner V, Kelsey SF, Olson $M$, et al. Insights from the NHLBI-Sponsored Women's Ischemia Syndrome Evaluation (WISE) Study: Part II: gender differences in presentation, diagnosis, and outcome with regard to genderbased pathophysiology of atherosclerosis and macrovascular and microvascular coronary disease. J Am Coll Cardiol. 2006;47 3 Suppl:S21-9.

3. Mieres JH, Bonow RO. Ischemic heart disease in women: a need for sex-specific diagnostic algorithms. JACC Cardiovasc Imaging. 2016;9:347-9.

4. Agrawal S, Van Eyk J, Sobhani K, Wei J, Bairey Merz CN. Myocardial Infarction, and the failure of risk scores in women. Journal of women's health. 2015;24:859-61.

5. Mieres JH, Gulati M, Bairey Merz N, Berman DS, Gerber TC, Hayes SN, et al. Role of noninvasive testing in the clinical evaluation of women with suspected ischemic heart disease: a consensus statement from the American Heart Association. Circulation. 2014;130:350-79.

6. Tailor TD, Kicska GA, Jacobs JE, Pampaloni MH, Litmanovich DE, Reddy GP. Imaging of Heart Disease in Women. Radiology. 2017;282:34-53.

7. Gulati M, Black HR, Shaw LJ, Arnsdorf MF, Merz CN, Lauer MS, et al. The prognostic value of a nomogram for exercise capacity in women. New Eng J Med. 2005;353:468-75.

8. Sanders GD, Patel MR, Chatterjee R, Ross AK, Bastian LA, Coeytaux RR, et al. Noninvasive technologies for the diagnosis of coronary artery disease in women: future research needs: identification of future research needs from comparative effectiveness review no 58 AHRQ future research needs papers. Rockville (MD). 2013.

9. Murthy VL, Naya M, Taqueti VR, Foster CR, Gaber M, Hainer J, et al. Effects of sex on coronary microvascular dysfunction and cardiac outcomes. Circulation. 2014;129:2518-27.

10. Taqueti VR, Hachamovitch R, Murthy VL, Naya M, Foster CR, Hainer J, et al. Global coronary flow reserve is associated with adverse cardiovascular events independently of luminal angiographic severity and modifies the effect of early revascularization. Circulation. 2015;131:19-27.

11. Shaw LJ, Raggi P, Schisterman E, Berman DS, Callister TQ. Prognostic value of cardiac risk factors and coronary artery calcium screening for all-cause mortality. Radiology. 2003;228:826-33. 
12. Makaryus AN, Sison C, Kohansieh M, Makaryus JN. Implications of gender difference in coronary calcification as assessed by ct coronary angiography. Clin Med Insights Cardiol. 2014;8 Suppl 4:51-5.

13. Tsang JC, Min JK, Lin FY, Shaw LJ, Budoff MJ. Sex comparison of diagnostic accuracy of 64-multidetector row coronary computed tomographic angiography: results from the multicenter ACCURACY trial. J Cardiovasc Comput Tomogr. 2012;6:246-51.

14. Yiu KH, de Graaf FR, Schuijf JD, van Werkhoven JM, Marsan NA, Veltman CE, et al. Age- and gender-specific differences in the prognostic value of CT coronary angiography. Heart. 2012;98:232-7.

15. Min JK, Dunning A, Lin FY, Achenbach S, Al-Mallah M, Budoff MJ, et al. Age- and sexrelated differences in all-cause mortality risk based on coronary computed tomography angiography findings results from the International Multicenter CONFIRM (Coronary CT Angiography Evaluation for Clinical Outcomes: An International Multicenter Registry) of 23854 patients without known coronary artery disease. Journal of the American College of Cardiology. 2011;58:849-60.

16. Koo BK, Erglis A, Doh JH, Daniels DV, Jegere S, Kim HS, et al. Diagnosis of ischemia-causing coronary stenoses by noninvasive fractional flow reserve computed from coronary computed tomographic angiograms, Results from the prospective multicenter DISCOVER-FLOW (Diagnosis of Ischemia-Causing Stenoses Obtained Via Noninvasive Fractional Flow Reserve) study. J Am Coll Cardiol. 2011;58:1989-97.

17. Patel MR, Peterson ED, Dai D, Brennan JM, Redberg RF, Anderson $\mathrm{HV}$, et al. Low diagnostic yield of elective coronary angiography. N Eng J Med. 2010;362:886-95.

18. de Silva R, Camici PG. Role of positron emission tomography in the investigation of human coronary circulatory function. Cardiovascular research. 1994;28:1595-612.

19. Lanza GA, Camici PG, Galiuto L, Niccoli G, Pizzi C, Di Monaco A, et al. Methods to investigate coronary microvascular function in clinical practice. J Cardiovasc Med. 2013;14:1-18.

20. Shome JS, Perera D, Plein S, Chiribiri A. Current perspectives in coronary microvascular dysfunction. Microcirculation. 2017;24.

21. Park SM, Merz CN. Women and ischemic heart disease: recognition, diagnosis and management. Korean Circulation Journal. 2016;46:433-42.

22. Mehta LS, Beckie TM, DeVon HA, Grines CL, Krumholz HM, Johnson MN, et al. Acute Myocardial infarction in women: a scientific statement from the american heart association. Circulation. 2016;133:916-47.

23. Boersma E. Primary coronary angioplasty vs. thrombolysis g. does time matter? a pooled analysis of randomized clinical trials comparing primary percutaneous coronary intervention and inhospital fibrinolysis in acute myocardial infarction patients. Eur Heart J. 2006;27:779-88.

24. Stone GW, Grines CL, Browne KF, Marco J, Rothbaum D, O’Keefe $\mathrm{J}$, et al. Comparison of inhospital outcome in men versus women treated by either thrombolytic therapy or primary coronary angioplasty for acute myocardial infarction. Am J Cardiol. 1995; 75:987-92.

25. Yu J, Mehran R, Grinfeld L, Xu K, Nikolsky E, Brodie BR, et al. Sex-based differences in bleeding and long term adverse events after percutaneous coronary intervention for acute myocardial infarction: three year results from the HORIZONS-AMI trial. Catheterization and cardiovascular interventions: official journal of the Society for Cardiac Angiography \& Interventions. 2015;85:359-68.

26. O'Donoghue M, Boden WE, Braunwald E, Cannon CP, Clayton TC, de Winter RJ, et al. Early invasive vs conservative treatment strategies in women and men with unstable angina and nonST-segment elevation myocardial infarction: a meta-analysis. JAMA. 2008;300:71-80.

27. Blomkalns AL, Chen AY, Hochman JS, Peterson ED, Trynosky $\mathrm{K}$, Diercks DB, et al. Gender disparities in the diagnosis and treatment of non-ST-segment elevation acute coronary syndromes: large-scale observations from the CRUSADE (Can Rapid Risk Stratification of Unstable Angina Patients Suppress Adverse Outcomes With Early Implementation of the American College of Cardiology/American Heart Association Guidelines) National Quality Improvement Initiative. J Am Coll Cardiol. 2005;45:832-7.

28. Gottlieb S, Harpaz D, Shotan A, Boyko V, Leor J, Cohen M, et al. Sex differences in management and outcome after acute myocardial infarction in the 1990s: A prospective observational community-based study, Israeli Thrombolytic Survey Group. Circulation. 2000;102:2484-90.

29. Radovanovic D, Erne P, Urban P, Bertel O, Rickli H, Gaspoz $J M$, et al. Gender differences in management and outcomes in patients with acute coronary syndromes: results on 20,290 patientsfrom the AMIS Plus Registry. Heart. 2007;93:1369-75.

30. Tra J, van der Wulp I, Appelman Y, de Bruijne MC, Wagner C. Adherence to guidelines for the prescription of secondary prevention medication at hospital discharge after acute coronary syndrome: a multicentre study. Netherlands Heart J. 2015;23:214-21.

31. Montalescot G, Sechtem U, Achenbach S, Andreotti F, Arden C, Budaj A, et al. 2013 ESC guidelines on the management of stable coronary artery disease. Eu Heart J. 2013;34:2949-3003.

32. Robinson JG, Wallace R, Safford MM, Pettinger M, Cochrane $B$, Ko MG, et al. Another treatment gap: restarting secondary prevention medications: the Women's Health Initiative. J Clin Lipidol. 2010;4:36-45.

33. Priori SG, Blomstrom-Lundqvist C, Mazznti A, Blom N, Borggrefe M, et al. 2015 ESC Guidelines for the management of patients with ventricular arrhythmias and the prevention of sudden cardiac death: The Task Force for the Management of Patients with Ventricular Arrhythmias and the Prevention of Sudden Cardiac Death of the European Society of Cardiology (ESC). Eur Heart J. 2015;36:2793-867.

34. Alam M, Bandeali SJ, Kayani WT, Ahmad W, Shahzad SA, Jneid H, et al. Comparison by meta-analysis of mortality after isolated coronary artery bypass grafting in women versus men. Am J Cardiol. 2013;112:309-17.

35. Petrie MC, Jhund PS, She L, Adlbrecht C, Doenst T, Panza JA, et al. ten-year outcomes after coronary artery bypass grafting according to age in patients with heart failure and left ventricular systolic dysfunction: an analysis of the extended follow-up of the stich trial (surgical treatment for ischemic heart failure). Circulation. 2016;134:1314-24. 